\title{
Response of Thermoelastic Beam due to Thermal Source in Modified Couple Stress Theory
}

\author{
Rajneesh Kumar \\ Department of Mathematics, Kurukshetra University Kurukshetra \\ Kurukshetra, India \\ E-mail: rajneesh_kuk@rediffmail.com
}

Received: 08 January 2016; revised: 14 April 2016; accepted: 26 April 2016; published online: 31 May 2016

\begin{abstract}
The present investigation deals with the problem of thermoelastic beam in the modified couple stress theory due to thermal source. The governing equations of motion for the modified couple stress theory and heat conduction equation for coupled thermoelasticity are investigated to model the vibrations in a homogeneous isotropic thin beam in a closed form by applying the Euler Bernoulli beam theory. The Laplace transform technique is used to solve the problem. The lateral deflection, thermal moment, axial stress average due to normal heat flux in the beam are derived and computed numerically. The resulting quantities are depicted graphically for a specific model. A particular case is also introduced.
\end{abstract}

Key words: thermoelastic beam, modified couple stress theory, classical coupled theory

\section{INTRODUCTION}

Cosserat and Cosserat [1] were the first to develop a mathematical model to analyze materials with couple stresses. In the original Cosserat theory, the kinematical quantities were the displacement and a material microrotation, which assumed to being independent of the continuum macrorotation. The couple-stress theory is an extended form of the continuum theory that includes the effects of a couple per unit area on a material volume, in addition to the classical direct and shear forces per unit area. Later on, Toupin [2] derived the associative constitutive equations for finite deformation of perfectly elastic materials and Mindlin and Tiersten [3] formulated a literalized theory of couple stress elasticity.

Yang et al. [4] modified the classical couple stress theory and proposed a modified couple-stress model, in which the couple stress tensor is symmetrical and only one material length parameter is needed to capture the size effect which is caused by micro-structure. The Bernoulli-Euler beam model based on a modified couple stress theory studied by Park and Gao [5]. Simsek and Reddy [6] investigated the bend- ing and vibration of functionally graded microbeams using a new higher order beam theory and the modified couple stress theory. Mohammad-Abadi and Daneshmehr [7] studied the size dependent buckling analysis of microbeams based on the modified couple stress theory with high order theories and general boundary conditions. Darijani and Shahdadi [8] investigated the effect of shear deformation on the static bending and vibration responses of a simply supported microplate by using the modified couple stress theory. Beni et al. [9] studied the size-dependent equations of motion for a functionally graded cylindrical shell on the basis of the modified couple stress theory. Dehrouyeh-Semnani et al. [10] studied the problems of microbeams based on the modified couple stress theory.

Sun et al. [11] used the Laplace transform technique to study the vibration phenomena due to pulsed laser heating of a microbeam under different boundary conditions. Thermoelastic beams with voids were studied by $\mathrm{Li}$ and Cheng [12]. Sharma [13] derived governing equations of flexural vibrations in a transversely isotropic, thermoelastic beam in a closed form based on the Euler-Bernoulli theory 
to study thermoelastic damping (TED) and frequency shift (FS) of vibrations in clamped and simply supported beam structures.

Zang and $\mathrm{Fu}$ [14] developed a new beam model for a viscoelastic micro-beam based on the modified couple stress theory. Ghader et al. [15] discussed the problem of thermoelastic damping in a micro-beam resonator using the modified couple stress theory. An eigenvalue formulation and the Galerkin finite element method used to evaluate the problem of thermoelastic damping in vented microelectromechanical systems (MEMS) beam resonators were presented by Guo et al. [16]. Simsek and Reddy [6] investigated the bending and vibration of functionally graded microbeams using a new higher order beam theory and the modified couple stress theory.

Abouelregal and Zenkour [17] discussed the problem of an axially moving microbeam subjected to sinusoidal pulse heating and an external transverse excitation with one relaxation time by using the Laplace transform and also studied the effects of the pulse- width of thermal vibration, moving speed and the transverse excitation. Sharma and Kaur [18] studied transverse vibrations in a thermoelastic-diffusive thin micro beam based on the Euler-Bernoulli theory under clampedclamped boundary conditions. Zenkour and Abouelregal [19] studied the problem of thermoelastic vibration of an axially moving microbeam subjected to sinusoidal pulse heating.

In this work, the response of a thermoelastic beam in the modified couple stress theory due to thermal source is investigated. A numerical technique based on the Laplace transformation is used to calculate the lateral deflection, thermal moment and axial stress average. The effect of couple stress on lateral deflection, thermal moment and axial stress average for coupled thermoelastic (CT) theory are shown graphically for a specific model. Particular cases of interest are also deduced from the present investigation.

\section{BASIC EQUATIONS}

Following Yang et al. [4] and Nowacki [20], the governing equations in a modified couple stress thermoelastic medium in the absence of body forces and heat sources are given by

(i) Constitutive relations

$$
\begin{gathered}
t_{i j}=\lambda e_{k k} \delta_{i j}+2 \mu e_{i j}-\frac{1}{2} e_{k i j} m_{l k, l}-\beta T \delta_{i j}, \\
m_{i j}=2 \alpha \chi_{i j}, \\
\chi_{i j}=\frac{1}{2}\left(\omega_{i, j}+\omega_{j, i}\right) \\
\omega_{i}=\frac{1}{2} e_{i p q} u_{q, p}, i, j, k=1,2,3 .
\end{gathered}
$$

(ii) Equations of motion

$$
\begin{aligned}
& \left(\lambda+\mu+\frac{\alpha}{4} \Delta\right) \nabla(\nabla . u) \\
+ & \left(\mu-\frac{\alpha}{4} \Delta\right) \nabla^{2} u-\beta \nabla T=\rho \ddot{u},
\end{aligned}
$$

(iii) Equation of heat conduction

$$
K \Delta T-\rho c_{e} \frac{\partial T}{\partial t}=T_{0} \beta \frac{\partial}{\partial t}(\nabla . u),
$$

where $t_{i j}$ are the components of stress tensor, $\lambda$ and $\mu$ are Lamé's constants, $\delta_{i j}$ is Kronecker's delta, $e_{i j}$ are the components of strain tensor, $e_{i j k}$ is alternate tensor, $m_{i j}$ are the components of couple-stress, $\beta=(3 \lambda+2 \mu) \alpha_{T}, \alpha_{T}$ is the coefficient of linear thermal expansion, $T$ is the temperature change, $\alpha$ is the couple stress parameter, $\chi_{i j}$ is symmetric curvature, $\omega_{i}$ is the rotational vector. $u=\left(u_{1}, u_{2}, u_{3}\right)$ is the components of displacement, $\rho$ is the density, $\Delta$ is the Laplacian operator, $\nabla$ is del operator. $K$ is the coefficient of the thermal conductivity, $c_{e}$ is the specific heat at constant strain, $T_{0}$ is the reference temperature assumed to be such that $T / T_{0} \ll 1$.

\section{FORMULATION OF THE PROBLEM}

We consider a homogeneous isotropic, rectangular modified couple stress thermoelastic beam of length $(0 \leq x \leq L)$, width $(-d / 2 \leq y \leq d / 2)$ and thickness $(-h / 2 \leq z \leq h / 2)$, where $x, y$ and $z$ are Cartesian axes lying along the length, width and thickness of the beam so that $x$-axis coincides with the beam axis and $y$ and $z$ axes coincide with the end $(x=0)$ with origin located at the axis of the beam.

Using the Euler-Bernoulli theory for small deflection of a simple bending problem, the components of displacement as

$$
\begin{aligned}
& u(x, y, z, t)=-z \frac{\partial w}{\partial x}, v(x, y, z, t)=0 \\
& w(x, y, z, t)=w(x, t)
\end{aligned}
$$

where $w(x, t)$ denotes the lateral deflection of the beam and $t$ denotes the time. The constitutive relation (1) in onedimension along the axis and with the help of equation (7), we obtain

$$
t_{x}=-(\lambda+2 \mu) z \frac{\partial^{2} w}{\partial x^{2}}-\beta M_{T},
$$

The bending moment resultant of the beam, $M$ can be evaluated via the following relation:

$$
M=M_{\sigma}+M_{m}=d\left(\int_{\frac{-h}{2}}^{\frac{h}{2}} t_{x} z d z+\int_{\frac{-h}{2}}^{\frac{h}{2}} m_{x y} d z\right)
$$


where $M_{\sigma}$ and $M_{m}$ are the components of the bending moment due to the classic stress and couple stress tensors, respectively.

Applying the Euler-Bernoulli assumption (7) and with the aid of equation (8) in (9), we obtain

$$
M=-(\lambda+2 \mu) I \frac{\partial^{2} w}{\partial x^{2}}-M_{T}-\alpha A \frac{\partial^{2} w}{\partial x^{2}},
$$

where $I$ is the second moment of the cross-section area of the beam and $M_{T}$ is the thermal moment and $I$ and $M_{T}$ are given by

$$
I=\int_{\frac{-h}{2}}^{\frac{h}{2}} d z^{2} d z=\frac{d h^{3}}{12}, M_{T}=\beta d \int_{\frac{-h}{2}}^{\frac{h}{2}} T z d z .
$$

The equation of transverse deflections of the beam is given by Rao [21]

$$
\frac{\partial^{2} M}{\partial x^{2}}-\rho A \frac{\partial^{2} w}{\partial t^{2}}=0
$$

where $A=d h$ is the cross-sectional area of the beam. From equations (10) and (12), yield

$$
[(\lambda+2 \mu) I+\alpha A] \frac{\partial^{4} w}{\partial x^{4}}+\frac{\partial^{2} M_{T}}{\partial x^{2}}+\rho A \frac{\partial^{2} w}{\partial t^{2}}=0,
$$

and the heat conduction equation can be written as

$$
\nabla^{2} T-\frac{\rho c_{e}}{K}\left(\frac{\partial T}{\partial t}\right)+\frac{\beta T_{0}}{K} z \frac{\partial^{3} w}{\partial x^{2} \partial t}=0
$$

Multiply (14) by $z d z$ and integrate from interval $(-h / 2, h / 2)$, yields

$$
\begin{gathered}
\left(\frac{\partial^{2} M_{T}}{\partial x^{2}}-\frac{12}{h^{2}} M_{T}\right)(x, t)+\frac{h \beta d}{2}\left\{\frac{\partial T}{\partial z}\left(x, \frac{h}{2}, t\right)\right. \\
\left.+\frac{\partial T}{\partial z}\left(x,-\frac{h}{2}, t\right)\right\}-\frac{\rho c_{e}}{K}\left(\frac{\partial M_{T}}{\partial t}\right)-\frac{\beta^{2} T_{0} I}{K} \frac{\partial^{3} w}{\partial x^{2} \partial t}=0
\end{gathered}
$$

where $M_{T}$ is mathematically approximated as the difference between the temperatures at the upper and bottom surfaces of the beam, the temperature is assumed to vary linearly through the thickness of the beam, thus we have

$$
T\left(x, \frac{h}{2}, t\right)-T\left(x,-\frac{h}{2}, t\right)=\frac{12}{\beta d h^{2}} M_{T}(x, t)
$$

The non-dimensional quantities can be written as

$$
\begin{aligned}
& x^{\prime}=\frac{x}{L} z^{\prime}=\frac{z}{L}, w^{\prime}=\frac{w}{L}, t^{\prime}=\frac{\nu t}{L}, T^{\prime}=\frac{\beta T}{E}, \\
& M^{\prime}=\frac{M}{d E h^{2}}, M_{T}^{\prime}=\frac{M_{T}}{d E h^{2}}, \nu^{2}=\frac{E}{\rho}, t_{x}^{\prime}=\frac{t_{x}}{E} .
\end{aligned}
$$

where $E=\frac{\mu(3 \lambda+2 \mu)}{(\lambda+\mu)}$ is the Young modulus, $\nu=\frac{\lambda}{2(\lambda+\mu)}$ is the Poisson ratio, respectively.
By using (17) in (13) and (15), after surpassing the primes, yield

$$
\begin{gathered}
\frac{\partial^{4} w}{\partial x^{4}}+a_{1} \frac{\partial^{2} M_{T}}{\partial x^{2}}+a_{2} \frac{\partial^{2} w}{\partial t^{2}}=0 \\
\left(\frac{\partial^{2} M_{T}}{\partial x^{2}}-12 a_{3}^{2} M_{T}\right)(x, t)+\frac{a_{3}}{2}\left\{\frac{\partial T}{\partial z}\left(x, \frac{h}{2}, t\right)\right. \\
\left.+\frac{\partial T}{\partial z}\left(x,-\frac{h}{2}, t\right)\right\}-a_{4} \frac{\partial M_{T}}{\partial t}+a_{5} \frac{\partial^{3} w}{\partial x^{2} \partial t}=0
\end{gathered}
$$

where

$$
\begin{aligned}
& a_{1}=\frac{d E h^{2} L}{(E I+\alpha A)}, \quad a_{2}=\frac{\rho A \nu^{2} L^{2}}{(E I+\alpha h)} \\
& a_{3}=\frac{L}{h}, \quad a_{4}=\frac{\rho c_{e} \nu L}{K}, \quad a_{5}=\frac{T_{0} \beta^{2} I \nu}{K d E h^{2}}
\end{aligned}
$$

\section{SOLUTION IN THE LAPLACE DOMAIN}

We define the Laplace transform as

$$
L\{f(t)\}=\int_{0}^{\infty} e^{-s t} f(t) d t=\bar{f}(s),
$$

where $s$ is the Laplace transform parameter. Applying Laplace transform defined by (20) on equations (18) and (19), we obtain

$$
\left(D^{4}+a_{2} s^{2}\right) \bar{w}=-a_{1} D^{2} \bar{M}_{T}
$$

$$
\left(D^{2}-\Gamma_{1}\right) \bar{M}_{T}+\Gamma_{2} D^{2} \bar{w}=-\bar{Q}(s),
$$

where

$$
\begin{aligned}
& D=\frac{d}{d x}, \Gamma_{1}=\left(12 a_{3}^{2}+a_{4} s\right), \Gamma_{2}=a_{5} s \\
& \bar{Q}(s)=\frac{a_{3}}{2}\left\{\frac{d \bar{T}}{d z}\left(x, \frac{h}{2}, s\right)+\frac{d \bar{T}}{d z}\left(x,-\frac{h}{2}, s\right)\right\} .
\end{aligned}
$$

The differential equation of the lateral deflection $\bar{w}$ and the thermal moment $\bar{M}_{T}$ are

$$
\left\{D^{6}-p D^{4}+q D^{2}-r\right\}\left[\begin{array}{l}
\bar{w} \\
\bar{M}_{T}
\end{array}\right]=\left[\begin{array}{c}
0 \\
-a_{2} s^{2} \bar{Q}(s)
\end{array}\right],
$$

where

$$
p=\left(\Gamma_{1}+a_{1} \Gamma_{2}\right), q=a_{2} s^{2}, r=q \Gamma_{1} .
$$

The differential equation governing the lateral deflection $\bar{w}$ can take the form

$$
\left(D^{2}-\lambda_{1}^{2}\right)\left(D^{2}-\lambda_{2}^{2}\right)\left(D^{2}-\lambda_{3}^{2}\right) \bar{w}=0,
$$

where $\pm \lambda_{1}, \pm \lambda_{2}$ and $\pm \lambda_{3}$ are the characteristic roots of the equation:

$$
\lambda^{6}-p \lambda^{4}+q \lambda^{2}-r=0
$$


and satisfy the well-known relations:

$\lambda_{1}^{2}+\lambda_{2}^{2}+\lambda_{3}^{2}=p, \lambda_{1}^{2} \lambda_{2}^{2}+\lambda_{2}^{2} \lambda_{3}^{2}+\lambda_{3}^{2} \lambda_{1}^{2}=q, \lambda_{1}^{2} \lambda_{2}^{2} \lambda_{3}^{2}=r$.

where $p, q, r$ are the sum of all the roots, sum of the roots taken two at a time and product of all the roots, respectively. Then the lateral deflection as:

$$
\bar{w}(x, s)=\sum_{i=1}^{3}\left(A_{i} e^{\lambda_{i} x}+B_{i} e^{-\lambda_{i} x}\right),
$$

where $A_{i}$ and $B_{i}, i=1,2,3$, are constant coefficients and depend on the Laplace variable $s$. The thermal moment is given by

$$
\bar{M}_{T}(x, s)=\sum_{i=1}^{3}\left(A_{i}^{\prime} e^{\lambda_{i} x}+B_{i}^{\prime} e^{-\lambda_{i} x}\right)+\frac{\bar{Q}}{\Gamma_{1}},
$$

where $A_{i}, A_{i}^{\prime}$ and $B_{i}, B_{i}^{\prime}, i=1,2,3$, are constant coefficients and depend on the Laplace variable $s$. Substituting (28) and (29) in (22), yields

$$
\left(\begin{array}{c}
A_{i}^{\prime} \\
B_{i}^{\prime}
\end{array}\right)=\frac{\Gamma_{2} \lambda_{i}^{2}}{\left(\Gamma_{1}-\lambda_{i}^{2}\right)}\left(\begin{array}{c}
A_{i} \\
B_{i}
\end{array}\right), \quad i=1,2,3 .
$$

In equation (29) and with the aid of (30), yield

$$
\bar{M}_{T}(x, s)=\sum_{i=1}^{3} \frac{\Gamma_{2} \lambda_{i}^{2}}{\left(\Gamma_{1}-\lambda_{i}^{2}\right)}\left(A_{i} e^{\lambda_{i} x}+B_{i} e^{-\lambda_{i} x}\right)+\frac{\bar{Q}}{\Gamma_{1}} .
$$

Making use of equations (8), (16), (17) and (20) and with the aid of (29) and (31), the axial stress is obtained

$$
\begin{aligned}
& \bar{T}_{x}(x, s)=\sum_{i=1}^{3} \lambda_{i}^{2}\left(\frac{-h(\lambda+2 \mu)}{E L}+\frac{12 \Gamma_{2}}{\left(\lambda_{i}^{2}-\Gamma_{1}\right)}\right) \\
& \times\left(A_{i} e^{\lambda_{i} x}+B_{i} e^{-\lambda_{i} x}\right)-\frac{12 \bar{Q}}{\Gamma_{1}},
\end{aligned}
$$

where

$$
\bar{T}_{x}(x, s)=\bar{t}_{x_{\mathrm{avg}}}(x, s)=\bar{t}_{x}\left(x, \frac{h}{2}, s\right)-\bar{t}_{x}\left(x,-\frac{h}{2}, s\right) .
$$

\section{APPLICATION}

We will discuss thermal loads over the upper surface of the beam:

The constant heat flux $\left(-q_{0}\right)$ normal to the upper surface $\left(z=\frac{h}{2}\right)$ of the beam and keeping bottom surface $\left(z=-\frac{h}{2}\right)$ at zero temperature gradient. The boundary conditions on the upper and bottom surfaces of heat conduction equation as

$$
q_{0}=K \frac{\partial T}{\partial z}\left(x, \frac{h}{2}, t\right), \frac{\partial T}{\partial z}\left(x,-\frac{h}{2}, t\right)=0 .
$$

Apply (17) and (20) on (34), we obtain

$$
\frac{d \bar{T}}{d z}\left(x, \frac{h}{2}, s\right)=\frac{q_{0}}{K}, \frac{d \bar{T}}{d z}\left(x,-\frac{h}{2}, s\right)=0 .
$$

\section{BOUNDARY CONDITIONS}

$$
\begin{aligned}
& w(0, t)=0, \quad \frac{\partial^{2} w(0, t)}{\partial x^{2}}=0, \quad M_{T}(0, t)=0 \\
& w(\mathrm{~L}, t)=0, \quad \frac{\partial^{2} w(\mathrm{~L}, t)}{\partial x^{2}}=0, \quad M_{T}(\mathrm{~L}, t)=0 .
\end{aligned}
$$

From (23) and (35), the thermal influence is given by

$$
\bar{Q}=\frac{a_{3} q_{0}}{2 K} \text {. }
$$

Using (17) and (20) in the boundary conditions (36) and (37), yield

$$
\begin{gathered}
\bar{w}(0, s)=0, \quad \frac{d^{2} \bar{w}(0, s)}{d x^{2}}=0, \quad \bar{M}_{T}(0, s)=0 \\
\bar{w}(1, s)=0, \quad \frac{d^{2} \bar{w}(1, s)}{d x^{2}}=0, \quad \bar{M}_{T}(1, s)=0 .
\end{gathered}
$$

Substituting the values of $\bar{w}$ and $\bar{M}_{T}$ from (28) and (31) in the boundary conditions (39) and (40), with the aid of (38), after some simplification, we obtain the expressions of lateral deflection, thermal moment and axial stress average as

$$
\begin{gathered}
\bar{w}(x, s)=\sum_{i=1}^{3}\left(A_{i} e^{\lambda_{i} x}+B_{i} e^{-\lambda_{i} x}\right) \\
\bar{M}_{T}(x, s)=\sum_{i=1}^{3} M_{i}\left(A_{i} e^{\lambda_{i} x}+B_{i} e^{-\lambda_{i} x}\right)+\frac{a_{3} q_{0}}{2 K \Gamma_{1}}, \\
\bar{T}_{x}(x, s)=\sum_{i=1}^{3} N_{i}\left(A_{i} e^{\lambda_{i} x}+B_{i} e^{-\lambda_{i} x}\right)-\frac{6 a_{3} q_{0}}{K \Gamma_{1}}
\end{gathered}
$$

where

$A_{1}, A_{2}, A_{3}, B_{1}, \mathrm{~B}_{2}, \mathrm{~B}_{3}$ are given in Appendix.

\section{PARTICULAR CASES}

(i) If $\alpha=0$, in equations (41)-(43), we obtain the results for lateral deflection, thermal moment and axial stress average in a thermoelastic beam and these results are similar as obtained by Sirafy et al. [22] in a specific case.

\section{Inversion of the Laplace transform}

To obtain the solution of the present application in the physical domain, we first apply the well-known formula:

$$
f(t)=\frac{1}{2 \pi i} \int_{c-i \infty}^{c+i \infty} \bar{f}(s) e^{-s t} d s,
$$




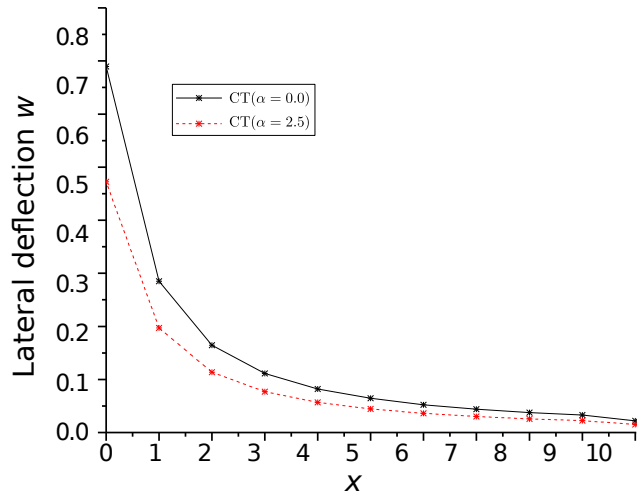

Fig. 1. Variation of lateral deflection $w$ with length $x$

Secondly, we adopt a numerical inversion method on the fourier series expansion, by which the integral (45) can be approximated as a series

$$
\begin{aligned}
& f(t)=\frac{e^{c t}}{t_{1}}\left[-\frac{1}{2} \operatorname{Re} \bar{f}(c)+\sum_{j=0}^{\infty} \operatorname{Re}\left(\bar{f}\left(c+\frac{i j \pi}{t_{1}}\right)\right)\right. \\
& \left.\times \cos \left(\frac{j \pi}{t_{1}}\right)-\sum_{j=0}^{\infty} \operatorname{Im}\left(\bar{f}\left(c+\frac{i j \pi}{t_{1}}\right)\right) \sin \left(\frac{j \pi}{t_{1}}\right)\right] \\
& -\sum_{j=1}^{\infty} \mathrm{e}^{-2 c j t_{1}} f\left(2 j t_{1}+t\right) .
\end{aligned}
$$

for $0 \leq t \leq 2 t_{1}$. The above series (45) is called the Durbin formula and the last term of this series is called the discretization error. Honig, U. Hirdes [23] developed a method for accelerating the convergence of the Fourier series and a procedure that computes approximately the best choice of the free parameters.

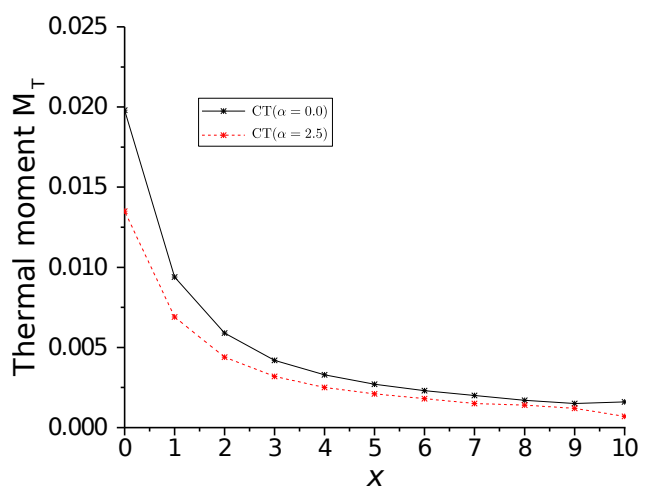

Fig. 2. Variation of thermal moment $M_{T}$ with length $x$

\section{NUMERICAL RESULTS AND DISCUSSION}

For numerical computations we take the magnesium material. The physical data chosen for magnesium are taken as Daliwal and Singh [24] and Sirafy et al. [22].

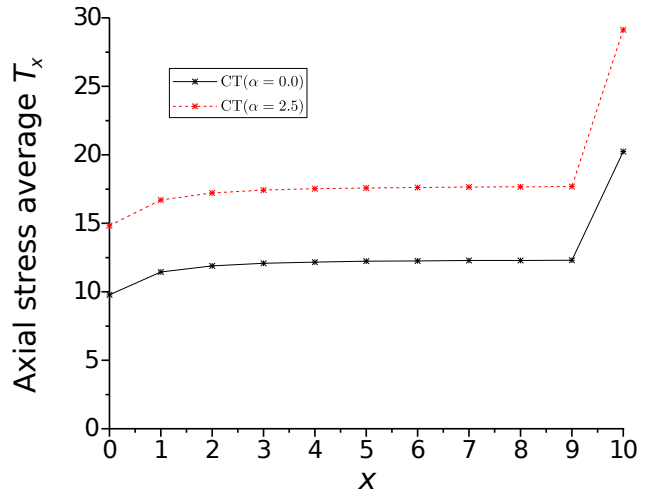

Fig. 3. Variation of axial stress average $T_{x}$ with length $x$

$$
\begin{aligned}
& \lambda=2.696 \times 10^{10} \mathrm{Kg} \mathrm{m}^{-1} \mathrm{~s}^{-2}, \\
& \mu=1.639 \times 10^{10} \mathrm{Kg} \mathrm{m}^{-1} \mathrm{~s}^{-2}, \\
& \rho=1.74 \times 10^{3} \mathrm{Kg} \mathrm{m}^{-3}, \\
& T_{0}=0.293 \times 10^{3} \mathrm{~K}, \\
& E=45 \times 10^{3} \mathrm{GPa}, \\
& \alpha_{t}=1.78 \times 10^{-5} \mathrm{~K}^{-1}, \\
& \nu=0.35, \\
& c_{e}=1.04 \times 10^{3} \mathrm{~J} \mathrm{Kg}^{-1} \mathrm{~K}^{-1}, \\
& q_{0}=2.25 \times 10^{11} \mathrm{Wm}^{-2}, \\
& L / h=10, d / h=0.5, h=10 \mu \mathrm{m}, \\
& \alpha=2.5 \mathrm{Kg} \mathrm{m} \mathrm{s}{ }^{-2}, \\
& K=1.7 \times 10^{2} \mathrm{Wm}^{-1} \mathrm{~K}^{-1}, \\
& t=0.5 \mathrm{~s} .
\end{aligned}
$$

The software Matlab 7.10.4 have been used to determine the absence and presence of couple stress on lateral deflection $w$, thermal moment $M_{T}$ and axial stress average $T_{x}$ for the coupled thermoelastic (CT) theory in the absence and presence of couple stress are computed numerically and shown graphically in Figs. 1-3, respectively. In all these Figs., the solid line with a centre symbol (-*-) corresponds to $\mathrm{CT}(\alpha=0)$, and the small dash line with a centre symbol $(--*--)$ corresponds to $\mathrm{CT}(\alpha=2.5)$.

Fig. 1 shows the variation of lateral deflection $w$ with respect to length. It is clear from the figure that the value of lateral deflection decreases monotonically in the considered region. Also, the value of lateral deflection for couple stress $(\alpha=2.5)$ is lower in comparison to $(\alpha=0)$ for the coupled thermoelastic theory.

Fig. 2 represents the variation of thermal moment $M_{T}$ with respect to length. It is evident that the value of thermal moment decreases as length increases further. On the other hand, the values of the thermal moment is higher in the absence of couple stress and smaller in the presence of couple stress for CT theory.

Fig. 3 depicts the variation of average of axial stress $T_{x}$ with respect to length. It is observed from the figure that the 
value of axial stress increases slowly in the range $0 \leq x \leq 9$ and then again increases abruptly in the remaining range. Similar behavior is observed for both cases of coupled thermoelastic $(\mathrm{CT})(\alpha=0,2.5)$.

\section{CONCLUSIONS}

Analysis of lateral deflection, thermal moment and axial stress average is a significant problem of solid mechanics. The resulting quantities are observed to be very sensitive towards the couple stress parameters. The interactions of a thermoelastic beam in the modified couple stress theory in the context of the coupled thermoelastic (CT) model of thermoelasticity has been investigated using the Euler-Bernoulli theory and the Laplace transform technique. A numerical technique has been used to recover the solutions in the physical domain. The expressions for lateral deflection, thermal moment and axial stress average have been derived successfully and shown graphically in the absence and presence of couple stress. It is observed from the figures that the value of lateral deflection, thermal moment are higher in the absence of couple stress and lower for presence of couple stress for the CT theory and reverse behavior is observed for axial stress average. The results obtained in the study should be beneficial for people working in medical science, thermomechanical, engineering, accelerometers, sensors, resonators and also working in the field of a thermoelastic beam in the modified couple stress theory for the coupled thermoelastic model.

\section{APPENDIX}

$$
A_{1}=\frac{\Delta_{1}}{\Delta}, A_{2}=\frac{\Delta_{2}}{\Delta}, A_{3}=\frac{\Delta_{3}}{\Delta}, \quad B_{1}=\frac{\Delta_{4}}{\Delta}, B_{2}=\frac{\Delta_{5}}{\Delta}, B_{3}=\frac{\Delta_{6}}{\Delta},
$$

and

$$
\begin{gathered}
\Delta=\left[\begin{array}{cccccc}
1 & 1 & 1 & 1 & 1 & 1 \\
e^{\lambda_{1}} & e^{\lambda_{2}} & e^{\lambda_{3}} & e^{-\lambda_{1}} & e^{-\lambda_{2}} & e^{-\lambda_{3}} \\
\lambda_{1}^{2} & \lambda_{2}^{2} & \lambda_{3}^{2} & \lambda_{1}^{2} & \lambda_{2}^{2} & \lambda_{3}^{2} \\
\lambda_{1}^{2} e^{\lambda_{1}} & \lambda_{2}^{2} e^{\lambda_{2}} & \lambda_{3}^{2} e^{\lambda_{3}} & \lambda_{1}^{2} e^{-\lambda_{1}} & \lambda_{2}^{2} e^{-\lambda_{2}} & \lambda_{3}^{2} e^{-\lambda_{3}} \\
M_{1} & M_{2} & M_{3} & M_{1} & M_{2} & M_{3} \\
M_{1} e^{\lambda_{1}} & M_{2} e^{\lambda_{2}} & M_{3} e^{\lambda_{3}} & M_{1} e^{-\lambda_{1}} & M_{2} e^{-\lambda_{2}} & M_{3} e^{-\lambda_{3}}
\end{array}\right], \\
M_{i}=\frac{\Gamma_{2} \lambda_{i}^{2}}{\left(\Gamma_{1}-\lambda_{i}^{2}\right)}, \quad N_{i}=\lambda_{i}^{2}\left(\frac{-h(\lambda+2 \mu)}{E L}+\frac{12 \Gamma_{2}}{\left(\lambda_{i}^{2}-\Gamma_{1}\right)}\right),
\end{gathered}
$$

$\Delta_{i}(i=1, \ldots, 6)$ are obtained by replacing $1^{\text {st }}, 2^{\text {nd }}, 3^{\text {rd }}, 4^{\text {th }}, 5^{\text {th }}$ and $6^{\text {th }}$ column by $\left[0,0,0,0,\left(-\frac{\bar{Q}}{\Gamma_{1}}\right),\left(-\frac{\bar{Q}}{\Gamma_{1}}\right)\right]^{T}$ in $\Delta_{i}$.

\section{References}

[1] E. Cosserat, F. Cosserat, Theory of deformable bodies. Hermann et Fils, Paris, 1909.

[2] R.A. Toupin, Elastic materials with couple-stresses, Arch. for Ratio. Mech. Analy. 11, 385-414 (1962).

[3] R.D. Mindlin, H.F. Tiersten. Effects of couple-stresses in linear elasticity, Arch. for Ratio. Mech. and Analy. 11, 415-448 (1962).

[4] F. Yang, A.C.M. Chong, D.C.C. Lam, P. Tong, Couple stress based strain gradient theory for elasticity, Int. J. Solids Struct. 39. 2731-43. (2002).

[5] S.K. Park, X.L. Gao, Bernoulli-Euler beam model based on a modified couple stress theory, J. of Micromech. and Micro Engg., 162355 (2006).

[6] M. Simsek, J.N. Reddy, Bending and vibration of functionally graded microbeams using a new higher order beam theory and the modified couple stress theory, Int. J. of Engg. Sci. 64, 37-53 (2013).

[7] M. Mohammad-Abadi, A.R. Daneshmehr, Size dependent buckling analysis of micro beams based on modified couple stress theory with high order theories and general boundary conditions, Int. J. of Engng. Sci 74, 1-14 (2014).

[8] H. Darijani, A.H. Shahdadi, A new shear deformation model with modified couple stress theory for microplates, Acta Mech. 226 2773-2788 (2015).

[9] Y.T. Beni, F. Mehralian, H. Razavi, Free vibration analysis of size-dependent shear deformable functionally graded cylindrical shell on the basis of modified couple stress theory, Composite Structures 120, 65-78 (2015).

[10] A.M. Dehrouyeh-Semnani M. Dehrouyeh, M. TorabiKafshgari, M. Nikkhah-Bahrami, A damped sandwich beam model based on symmetric-deviatoric couple stress theory, Int. J. of Engng. Sci. 92, 83-94 (2015). 
[11] Y. Sun, D. Fang, M. Saka, A.K. Soh, Laser-induced vibrations of micro-beams under different boundary conditions, Int. J. of Solids and Structures 45, 1993-2013 (2008).

[12] Y. Li, C.J. Cheng, A nonlinear model of thermoelastic beams with voids, with applications $\mathrm{J}$. of mech. of materials and Structures 5(5), 805-820 (2010).

[13] J.N. Sharma, Thermoelastic damping and frequency shift in Micro/Nano-Scale anisotropic beams, J. of Thermal Stresses 34, 650-666 (2011).

[14] J. Zang, Y. Fu, Pull-in analysis of electrically actuated viscoelastic microbeams based on a modified couple stress theory, Meccanica 47, 1649-1658 (2012).

[15] G. Rezazadeh, A.S. Vahdat, S. Tayefeh-Rezaei, C. Cetinkaya, Thermoelastic damping in a micro-beam resonator using modified couple stress theory, Acta Mechanica 223(6), 1137-1152 (2012).

[16] X. Guo X, Y.B. Yi, S. Pourkamali, A finite element analysis of thermoelastic damping in vented MEMS beam resonators, Int. J. of Mech. Sci. 4: 73-82 (2013).

[17] A.E. Abouelregal, A.M. Zenkour, Effect of phase lags on thermoelastic functionally graded microbeams subjected to ramp-type heating, Iranian Journal of Science and Technology: Transactions of Mechanical Engineering 38(M2), 321-335 (2014).

[18] J.N. Sharma, M. Kaur, Transverse vibrations in thermoelasticdiffusive thin micro-beam resonators, J. of Thermal Stresses 37, 1265-1285 (2014).

[19] A.M. Zenkour, A.E. Abouelregal, Thermoelastic Vibration of an Axially Moving Microbeam Subjected to Sinusoidal Pulse Heating, Int. J. Str. Stab. Dyn. 15(6), 1-15 (2015).

[20] W. Nowacki, Dynamical problems of thermo diffusion in solids, Engg. Frac. Mech. 8, 261-266 (1976).

[21] S.S. Rao, Vibrations of continuous systems. John Wiley \& Sons, New York 2007.

[22] I.H. EI-Sirafy, M.A. Abdou, E. Awad, Generalized lagging response of thermoelastic beams, Mathematical Problems in Engineering Article ID 780679, 1-13 (2014).

[23] G. Honig, U. Hirdes, A method for the numerical inversion of the Laplace transform, J. Comput. Appl. Math. 10, 113-132 (1984).

[24] R.S. Daliwal, A. Singh Dynamical coupled thermoelasticity. Hindustan Publishers, Delhi, 1980.

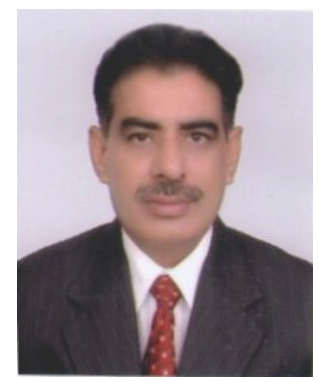

Rajneesh Kumar is a professor at Kurukshetra University Kurukshetra (Haryana, India). He received his PhD in Applied Mathematics from Guru Nanak Dev University, Amritsar (Punjab, India) in 1986. His area of interests include Continuum Mechanics (Micropolar elasticity, thermoelasticity, poroelasticity, magnetoelasticity, micropolar porous couple stress theory, viscoelasticity, double porosity, modified couple stress theory. He has published 448 publications in international journals. 